Published in final edited form as:

Curr Psychiatry Rep. 2013 December ; 15(12): 426. doi:10.1007/s11920-013-0426-8.

\title{
Use of Antipsychotic Medications in Pediatric Populations: What Do the Data Say?
}

\author{
Robert B. Penfold, PhD, \\ Group Health Research Institute and Department of Health Services Research, University of \\ Washington
}

Christine Stewart, MA,

Group Health Research Institute

Enid M. Hunkeler, MA,

Kaiser Permanente Division of Research, Northern California

Jeanne M. Madden, PhD,

Harvard Pilgrim Health Care Research Institute and Department of Population Medicine, Harvard University

Janet Cummings, PhD,

Department of Health Policy and Management, Rollins School of Public Health Emory University

Ashli A. Owen-Smith, PhD,

Kaiser Permanente Center for Health Research, Georgia

Rebecca C. Rossom, MD,

Health Partners Institute for Education and Research and Department of Psychiatry, University of Minnesota

Christine Lu, PhD,

Harvard Pilgrim Health Care Research Institute and Department of Population Medicine, Harvard University

Frances L. Lynch, PhD,

Kaiser Permanente Center for Health Research, Northwest

Beth E. Waitzfelder, PhD,

Kaiser Permanente Center for Health Research, Hawaii

Karen A. Coleman, PhD,

Kaiser Permanente Center for Health Research, Southern California

Robert B. Penfold, Group Health Research Institute and Department of Health Services Research, University of Washington, 1730 Minor Ave., Suite 1600, Seattle, WA 98101, (206) 287-2232 voice, (206) 287-2871 fax, Penfold.r@ ghc.org.

Compliance with Ethics Guidelines: Conflict of Interest: Robert B. Penfold, Christine Stewart, Janet Cummings, Ashli A. OwenSmith, Rebecca C. Rossom, Christine Lu, Frances L. Lynch, Beth E. Waitzfelder, and Arne L. Beck declare that they have no conflict of interest.

Enid M. Hunkeler has patent applications pending for an individualized health care management system and a computer-implemented method for assisting a care partner in monitoring a patient with a chronic disease.

Human and Animal Rights and Informed Consent: This article does not contain any studies with human or animal subjects performed by any of the authors. 
Brian K. Ahmedani, PhD, Henry Ford Health System

Arne L. Beck, PhD,

Kaiser Permanente Institute for Health Research, Colorado

John E. Zeber, PhD, and

Center for Applied Health Research, Scott and White Healthcare

Greg E. Simon, MD, MPH

Group Health Research Institute and Department of Psychiatry, University of Washington

\section{Abstract}

Recent reports of antipsychotic medication use in pediatric populations describe large increases in rates of use. Much interest in the increasing use has focused on potentially inappropriate prescribing for non FDA-approved uses and use amongst youth with no mental health diagnosis. Different studies of antipsychotic use have used different time periods, geographic and insurance populations of youth, and aggregations of diagnoses. We review recent estimates of use and comment on the similarities and dissimilarities in rates of use. We also report new data obtained on 11 Health Maintenance Organizations that are members of the Mental Health Research Network in order to update and extend the knowledge base on use by diagnostic indication. Results indicate that most use in pediatric populations is for disruptive behaviors and not psychotic disorders. Differences in estimates are likely a function of differences in methodology; however, there is remarkable consistency in estimates of use by diagnosis.

\section{Keywords}

Antipsychotics; Children; Adolescents; Medicaid; Mental Health Research Network; Off-label; MarketScan; IMS Health; NAMCS; NDTI; NCS-A

\section{Introduction}

Prescriptions for antipsychotic medications among children are reported to have increased greatly in recent years [1,2]. Most antipsychotic medications prescribed to children are "second generation" or "atypical" antipsychotic medications. These medications are argued to have milder side effect profiles (e.g., reduced extrapyramidal symptoms) than "first generation" or "typical" antipsychotic medications. However, the side effects of second generation antipsychotics (SGAs) can still be severe [3, 4]. The side effect profiles of individual medications in the same class differ significantly and their impact on the individual patients who take them are also known to differ [5-7]. Of the ten SGAs approved by the Food and Drug Administration (FDA) for adults in the United States, four of these (risperidone, olanzapine, quetiapine, and aripiprazole) are approved as treatments for pediatric bipolar disorder (ages 10 to 17) and schizophrenia (ages 13 to 17). Only risperidone and aripiprazole are approved for the treatment of irritability in children with autism spectrum disorders (ASD); risperidone is approved for ASD patients aged 5 to 16 years and aripiprazole for those aged 6 to 17 . 
Much scholarly research has focused on potentially inappropriate prescribing for non FDAapproved uses. For example, these medications have been prescribed for children, who exhibit disruptive behaviors and aggression $[8,9]$ but who do not have any of the diagnoses for which the FDA has approved their use. Most antipsychotic medication use among children and adolescents is "off label", that is, for indications that are not approved by the FDA or used for children who are younger than the approved age ranges [10-14]. Such uses include (but are not limited to): sleep disorders, Attention Deficit Hyperactivity Disorder, Oppositional Defiant Disorder, Conduct Disorder, Major Depressive Disorder, and PostTraumatic Stress Disorder. SGAs are also prescribed to individuals with developmental delay to treat aggression and self-injurious behaviors [15-18]. Lack of FDA approval stems in part from the ethics of protecting vulnerable populations from clinical trials research during drug development. The risk of harm to children and adolescents, particularly in the developmental phase of new medications, deters drug developers from including pediatric populations in research. Also, drug developers have limited financial incentives to conduct safety and efficacy trials of antipsychotic medications among children because clinicians are prescribing the medications in the absence of pediatric-specific evidence. Pediatric RCTs usually lag availability by years, if they are conducted at all. By the time the results of such trials are known, clinical practice has become entrenched and the impact of the research on prescribing patterns is minimal - even when the newest and most expensive medications are found to be no more effective and no safer than older, cheaper medications.

Considering the increasing overall prevalence of off-label SGA use in children and adolescents, the goal of this study was to review recent research concerning use in this population by diagnostic category and to compare and contrast this use by indication across different population estimates. We were particularly interested in comparing estimates of use for approved and non-approved uses and use among children and adolescents with no apparent mental health diagnosis. We also present new estimates of SGA use in a population of managed care enrollees across 11 sites in the Mental Health Research Network.

\section{Methods}

We conducted a review of recently published studies regarding the prevalence of SGA use in pediatric populations. We searched Medline using the key words: antipsychotic and (pediatric or children or adolescent). We also searched on the generic name of each SGA approved by the FDA and pediatric, children, or adolescent. The publication date was limited to be between January 2010 and August 2013. Our search yielded 6 studies reporting prevalence of use of antipsychotic medications by diagnostic category in children and adolescents. We provide a critical review of the methods and populations of patients included in these papers.

We also conducted a descriptive analysis of SGA utilization in 11 managed care organizations that are members of the Mental Health Research Network - a subset of organizations who are members of HMO Research Network and funded by the National Institute of Mental Health (NIMH). We compared the rates of diagnoses within groups of patients using classes of psychotropic medications. These data offer new insights into rates 
of off-label use by diagnosis including use among children and adolescents with no apparent diagnosis.

\section{Population}

The new data reported here are derived from the pharmacy claims data across the MHRN. These data are harmonized using a common data model so as to be in the same format at each site. The harmonized data are known as the Virtual Data Warehouse.

The MHRN rates reported are mental health diagnoses for all children aged less than 18 years as of December 31, 2011 who filled at least one prescription for a second generation antipsychotic medication in 2011. Individuals were required to have at least 10 months of continuous enrollment in a health plan that included prescription drug coverage at one of the MHRN sites. A single International Classification of Diseases, $9^{\text {th }}$ Revision, Clinical Modification (ICD9-CM) diagnosis code for any mental health condition (290 - 319) was counted as evidence of a diagnosis. All diagnosis codes in 2011 were counted. Individuals could have had more than one diagnosis and the corresponding rates of diagnoses among children and adolescents using SGAs sum to more than 100 percent.

The total population of individuals meeting the enrollment criteria includes 258,597 individuals aged 0-5 years, 294,722 aged 6-11 years, and 331,388 aged 12-17 years. To provide a comparison to adult rates of diagnoses among SGA users, we also report on $3,767,365$ individuals aged greater than 18 years.

\section{Review of Data on Antipsychotic Utilization in Pediatric Populations}

Previous estimates of antipsychotic medication use rates generally fall into three categories - those based on Medicaid administrative claims, those based on commercial administrative claims data (e.g., Truven Health Analytics, Marketscan), and those based on nationally representative surveys of patients and clinicians. Each of these data sources has strengths and limitations.

\section{Medicaid-based Estimates}

A 2009 study by Crystal and colleagues [19] estimated rates of antipsychotic medication use with Medicaid Analytic Extract Data (MAX) for seven states (CA, FL, GA, IL, NY, OH, and TX) in 2004. The study included continuously enrolled children and adolescents aged 6 to 17 years. Use was defined as the occurrence of at least one prescription claim for an antipsychotic medication and reported among those aged 6-12 and 13-17. Mental health diagnoses were obtained from inpatient and outpatient MAX files. All claims for an individual in the MAX data can be linked by a unique Medicaid Statistical Information System (MSIS) identification number.

The overall rate of use in this sample of youth was 4.2 percent $(n=88,096)$. Of those using antipsychotic medication, about 3.3\% had a diagnosis of schizophrenia and $18.7 \%$ had a diagnosis of bipolar disorder. Other notable prevalent diagnoses included ADHD (29.1\%), 
conduct disorder or disruptive behavior disorder without ADHD (8.9\%), anxiety or depression (9.1\%) and Autism (4.9\%). Roughly 9.1\% had no mental health diagnosis.

The 2011 study by dos Reis and colleagues [20] reported on antipsychotic use among children in Foster Care and insured by Medicaid in a mid-Atlantic state. The data in this study were from 2003 and identified 16,969 individuals aged less than 20 years who were continuously enrolled, had a mental health diagnosis, and at least one prescription claim for an antipsychotic. Unlike the study by Crystal and colleagues[19], dos Reis and co-authors required two clinical encounters with a mental health diagnosis in order for the diagnosis to be considered valid. Also, the dos Reis study excluded cases where a prescription occurred but no mental health diagnosis was found.

The overall use of antipsychotics in this foster care population was $2.7 \%$. Only $5 \%$ of youth using antipsychotics had a diagnosis of schizophrenia and $21 \%$ had a diagnosis of bipolar disorder. Over $53 \%$ of antipsychotic users had a diagnosis of ADHD, 33.8\% Depression, 26.3\% Conduct Disorder, 26.8\% Oppositional Defiant Disorder, and 5.4\% Autism Spectrum Disorder.

Finally, a 2012 study by Matone and colleagues [21] reported rates of antipsychotic medication use with MAX data from 50 states for the years 2002 through 2007. This comprehensive study included 15.2 million children aged 3 to 18 years with at least 10 months of continuous eligibility in each year. Youth were divided into 3 age categories (3-5, 6-11, and 12-18) and 10 diagnostic categories. Like other studies, a single diagnosis code was considered valid evidence of a mental health condition.

Of the 3.55 million children aged 3-5 years, the overall rate of SGA use was 0.4 percent in 2007. The corresponding rates were $2.1 \%$ in youth $6-11(\mathrm{n}=5.83$ million $)$ and $3.7 \%$ in adolescents $12-18(\mathrm{n}=5.78$ million). Detailed rates were also reported by age and diagnostic category. Among children aged 3-5 years in 2007 using SGAs, 0.93\% had a diagnosis of bipolar disorder alone (no comorbid mental health diagnoses), and none were diagnosed with schizophrenia. Across other diagnoses, 13.8\% had a diagnosis of ADHD alone, $5.5 \mathrm{had}$ a diagnosis of conduct disorder alone, and $4 \%$ had a diagnosis of Autism alone. Over 25\% had 3 or more comorbid mental health diagnoses and $11.3 \%$ had no mental health diagnoses.

In youth aged 6-11 using antipsychotics, a diagnosis of schizophrenia appeared in $0.05 \%$ of cases and Bipolar Disorder in $1.8 \%$ of cases. A similar pattern of diagnoses was reported in youth aged 6-11 for other diagnoses with $19.6 \%$ of youth in this category having a diagnosis of ADHD, 3.7\% Conduct Disorder, and 2.2\% Autism. About 8.6\% had a diagnosis of ADHD and Conduct Disorder together. Over $26 \%$ had 3 or more comorbid mental health conditions and $10.1 \%$ had no mental health diagnosis.

The distribution of diagnoses in youth aged 12-18 years was similar to that for those aged 6-11 with $0.4 \%$ of users having a diagnosis of schizophrenia and $4 \%$ having a diagnosis of Bipolar Disorder. Other select diagnoses included ADHD (10.5\%), Conduct Disorder (4.4\%), and Autism (1.5\%). Over 29.4\% had 3 or more comorbid diagnoses and $13 \%$ had no mental health diagnoses. Table 1 provides estimates of antipsychotic medication use. 


\section{Strengths and Limitations of the Medicaid-based Estimates}

One strength of the Medicaid-based rates is the large sample size used to generate estimates, which results in high precision. The multi-state studies are also important because a large number of children are insured by Medicaid in the U.S., and these studies therefore have important policy implications for Medicaid budgets. Estimating rates for economically disadvantaged children and those at increased risk of mental health problems (e.g., children in foster care) is also important in order to evaluate potentially inappropriate use (e.g., use of antipsychotics rather than psychotherapy).

There are several limitations to the Medicaid-based rate estimates. First, it is not possible to determine the validity of the diagnoses (e.g., through chart review or structured clinical interview) for such a large population of youth. Diagnoses from primary care clinicians and mental health specialists likely have different positive predictive values. MAX data are often missing the rendering provider number and, even when present, it is necessary to contact states individually to obtain data on providers. In addition, access to mental health specialists has been a significant concern for children covered by Medicaid [22-25], making the likeliness of diagnosis by a provider that is not a mental health specialist more likely.

It is also not possible to determine the exact mental health disorder for which the antipsychotic medication was prescribed. In cases where there are multiple psychiatric diagnoses (e.g., Bipolar Disorder and ADHD) it is impossible to determine whether the prescription might be inappropriate. Further, none of the studies reported on the quantity of medication dispensed, so it is not possible for example to distinguish ongoing use of the medications from short-term acute use in the reported results. Ongoing use is more likely to be associated with the emergence of negative side effects (e.g., weight gain).

It is also difficult to reconcile the differences in diagnostic rates across the various estimates without standardizing the populations with respect to age, sex, race, and eligibility status (e.g., poverty, SSI, foster care), and managed care. For example, the Crystal [19] study used only 7 states and reported on those aged 6-17 whereas Matone [21] reported on those aged 3-18 in 50 states. Differences in the composition of the populations are likely responsible for some of the differences in estimated rates.

Finally, the MAX data are almost exclusively claims for providers billing Medicaid on a feefor-service (FFS) basis. However, the majority of children insured by Medicaid in the U.S. are enrolled in managed care plans where a health maintenance organization (HMO) receives capitated payments for enrollees. The FFS data likely capture claims for individuals who are more difficult to enroll in managed care plans such as those with family and/or housing instability. It is possible that the MAX data are biased towards a population of youth with a higher disease burden and greater use of pharmacotherapy and less access to psychotherapy.

\section{Pediatric Utilization Rates Estimated From Commercial Data}

Alongside the Medicaid-based estimates, Crystal [19] reported on rates using 2006

Marketscan data from Truven Health Analytics. The Marketscan data include administrative

Curr Psychiatry Rep. Author manuscript; available in PMC 2014 December 01. 
claims information from a variety of Commercial payers as well as Medicaid. In these data, limited to privately insured youth, the overall antipsychotic utilization rate in children and adolescents was $0.21 \%$. Of these users, $2.2 \%$ had a diagnosis of Schizophrenia, 25.2\% Bipolar Disorder, 21.4\% ADHD, 16\% anxiety or depression, 4.5\% Conduct Disorder or Disruptive Behavior Disorder, and 5.2\% Autism. About 14.6\% had no mental health diagnosis.

\section{Strengths and limitations of the Commercial Data}

Strengths of the Marketscan data include the large sample size and national representativeness of the population of Americans with employer-provided health insurance and Medicaid. Marketscan data also include inpatient medication dispensings which Medicaid pharmacy claims do not (although some of these data are derived from a proprietary projection methodology of unknown quality). Like the Medicaid data, diagnoses are not validated through chart review. Also, the data derived from large employers so small and medium sized firms are not represented. Finally, individuals (and their dependent children) in the Marketscan data have a unique identifier for every employer. Individuals can be easily followed over time so long as primary insured person does not change employers. If the primary insured person moves to a different employer for whom Truven Analytics collects data then they are assigned a new identifier. Thus, an unknown amount of duplication in the rates of diagnosis and utilization occurs. The amount of duplication is likely to be small as a proportion of all claims; however, the effect of double counting may be greater for less common diagnoses (e.g., schizophrenia, bipolar disorder).

\section{Survey-based Data}

Alexander and colleagues 2011 study [14] reported on antipsychotic medication use between 1995 and 2008 using physician survey data from IMS Health. The National Diagnostic and Therapeutic Index (NDTI) data are generated from a stratified random sample of approximately 4,800 office-based physicians who report on two consecutive workdays per quarter. Respondents fill out an encounter form that details patients' diagnoses and medications prescribed. The authors reported on all visits where an antipsychotic medication was prescribed. Using the diagnosis at the same visit where the prescription occurred, the authors classified the evidence in support of that indication as either "on label", off-label with moderate or strong evidence, or off-label with uncertain evidence using the Drugdex drug compendium. The distribution of diagnoses was reported for all individuals but not separately for youth. Nevertheless, the results regarding on-label and off-label use are informative. Of the 4,216,000 individuals aged less than 18 years using SGAs in 2008, 67\% had an off-label indication with uncertain evidence and $9 \%$ had an indication with moderate or strong evidence. Only $24 \%$ of use in this cohort was on-label.

Olfson and colleagues 2012 study [2] reported on antipsychotic use rates using National Ambulatory Care Medical Survey (NAMCS) data. Data across the 2005-2009 time periods were combined to stabilize estimates. The NAMCS data are nationally representative of visits to physicians in office-based settings. The physician or staff member reports information on visits and the authors report on those visits at which an antipsychotic 
prescription occurred. The weighted numerators for calculating rates are based on 270 actual visits for children and 257 actual visits for adolescents. The denominator for calculating rates of pediatric use was estimated from U.S. Census Bureau data.

Of the children aged 0-13 included in this sample, $6 \%$ had a diagnosis of Schizophrenia and $12.2 \%$ had a diagnosis of Bipolar Disorder. Over 63\% had a diagnosis of disruptive behavior disorder. About $12 \%$ had no mental health diagnosis. The authors reported that $94 \%$ of use in this population had no approved indication. The distribution of rates in adolescents aged 14-20 years were somewhat similar with $8.1 \%$ having a diagnosis of Schizophrenia and $28.8 \%$ having a diagnosis of Bipolar Disorder. Roughly $34 \%$ of individuals in this age group had a diagnosis of disruptive behavior disorder and $14.8 \%$ had no diagnosis.

The final study identified was by Merikangas and co-authors in 2013 [26]. The investigators used data from the National Comorbidity Survey, Adolescent Supplement (NCS-A). These nationally representative data were collected on 10,123 youth aged 13-18 years between February 2001 and January 2004. Unlike the claims-based or visit-based estimates reviewed here, the NCS-A estimates were based on structured interviews conducted by professional (but lay) interviewers. Computerized algorithms were used to assign Diagnostic and Statistical Manual of Mental Disorders, Fourth Edition (DSM-IV) diagnoses. However, in contrast to the other studies reviewed here, medication use was self-reported over the previous 12 months.

The rates reported in the Merikangas study[26] are not directly comparable to other data reviewed here because the rates are antipsychotic use among those diagnosed rather than the diagnoses among those using antipsychotics. Among those with a developmental disorder (e.g., Autism), 7.6\% reported using an antipsychotic (the confidence interval; however, was $2.3 \%-21.9 \%$ ). Of those with a diagnosis of Bipolar Disorder 2.6\% reported antipsychotic use; and of youth with ADHD, $1.5 \%$ reported antipsychotic use. Only $0.1 \%$ of those with no mental health diagnosis reported using an antipsychotic. Schizophrenia was not assessed.

In a companion paper, Olfson and Merikangas [27] reported on the diagnostic characteristics of the 51 children reporting use of antipsychotics in the NCS-A data. Of users, $59.1 \%$ had any behavior disorder, $38.3 \%$ had any anxiety disorder, and $24.8 \%$ had any mood disorder.

\section{Strengths and limitations of the Survey-based data}

While the results of the Alexander study appear to be generally similar to the rates reported using Medicaid data, there are limitations to the NDTI-based estimates. First, the authors extrapolated the national estimates from the sample data for visits by patients of all ages [14]. And while the authors cite previous studies of the comparability of the NDTI and National Ambulatory Care Medical Survey (NAMCS), it is not clear that the NDTI is representative of mental health care or antipsychotic prescriptions. Aside from the statistical methodology, the estimates are also based on visits and particularly on visits where the diagnosis and prescription occurred together. Like the NDTI-based estimates, the NAMCSbased estimates are visit-based rather than patient based. Diagnoses from other clinicians (e.g., consulting psychiatrist) or from previous visits are missing. There is also no diagnostic information from inpatient settings available. Further, Olfson and colleagues [2] 
acknowledge that they cannot derive unique patients and an unknown amount of patient duplication occurs in the estimates.

Encounter reports completed by physicians likely do not capture ongoing medication use prescribed by other clinicians or diagnoses given by other clinicians at separate encounters contemporaneously or in the recent past prior to the index encounter.

\section{Mental Health Research Network Data}

Recent estimates of antipsychotic use in youth reviewed above are based on somewhat old data. This study was conducted in part to provide updated estimates on the use of these medications. Table 2 provides detailed estimates of diagnoses among children, adolescents and adults who used SGAs in 2011.

What is striking about the MHRN rates is many youth using SGAs have disruptive behavior disorders, attention disorders, and mood disorders. These diagnoses are frequently comorbid with Bipolar Disorder and Autism Spectrum Disorders. The absence of a mental health diagnosis among antipsychotic users is also notable because they are much lower than in the studies reviewed above. Only $8.1 \%$ of females aged 6-11 and $4.4 \%$ of female users aged 12-17 had no mental health diagnosis. Among males, the comparable rates were 5.6\% and $6.8 \%$.

The MHRN data have limitations as well. The HMO population of children is more likely to be composed of dependents of employed parents. And similar to any claims-based analysis, the diagnoses could not be individually validated due to the large number of youth.

\section{What Do the Data Say?}

Despite the demographic differences in study populations and methodologic difficulties with claims-based and visit-based approaches to estimating rates of antipsychotic use, some important conclusions can be drawn by assessing similarities in the results reported. First, it is clear that the vast majority of antipsychotic medication use in children and adolescents is for disruptive behavior. Only a small percentage, perhaps 3-4\%, of antipsychotic use appears to be for children with schizophreniform disorders or psychoses. This is not terribly surprising considering that these disorders are much less prevalent than attention disorders and mood disorders.

It is also clear that there is a heavy burden of comorbid mental health problems in youth using antipsychotic medications. One striking example is in the MHRN population of male users aged 6-11 years. In this group, 66.9\% had an attention disorder, $28.5 \%$ had an anxiety disorder, $32 \%$ had an autism spectrum disorder, $43.1 \%$ had a disruptive behavior disorder, and $31.5 \%$ had another mood disorder. Another interpretation of these data is that there is a large degree of diagnostic uncertainty in children and adolescents with behavioral health problems. The specific diagnosis in each child or adolescent using an antipsychotic medication may not be clear and individuals may therefore accrue diagnoses such as ADHD and conduct disorder before ultimately being diagnosed with schizophrenia or bipolar 
disorder. More research is needed to identify the degree to which disruptive behaviors are prodromal symptoms of serious mental illness.

Where the estimates differ is likely attributable to differences in methodology. The large claims-based samples offer stronger evidence of prescription use than self-reported use because a prescription claim record exists, but the validity of the diagnoses for these youth is unknown. In contrast, estimates based on NCS-A data have high diagnostic validity and weaker evidence of antipsychotic use. The self-reported use of medication over the last year likely underestimates use. In a study by Simon and colleagues [28], people queried about the effectiveness of their antidepressant could not accurately recall their treatment response as compared to their depression scores in the medical record, even after it was verified that the patient filled the prescription and the patient was shown a picture of the pill. Other evidence on medication recall is mixed, with some studies reporting good recall compared to pharmacy records and others poor agreement [29-32]. We are somewhat more confident in the rates of antipsychotic use derived from claims-based data.

Another important difference in the estimates of antipsychotic utilization rates is that the NCS-A study diagnosed youth at home and in school settings; not in a health care setting. In contrast, not all youth in the NCS-A study were engaged with the health care system for behavioral health problems.

These data cannot reliably be used to comment on the appropriateness of antipsychotic medication use. Without detailed chart review, we cannot know from the claims data or visit-based data what symptoms the clinicians were trying to treat. In addition, we cannot be certain of the original setting of diagnoses (e.g., primary care consultation with psychiatry), or whether the patient had tried previous medications that were ineffective. Future studies using detailed chart reviews of electronic medical records or surveys of providers prescribing these medications might facilitate valid studies of appropriateness in the future; however, these analyses would be very expensive to conduct.

\section{Conclusion}

All the estimates of antipsychotic use among youth follow a similar distribution of diagnoses regardless of the populations studied. The data support the conclusion that most use is not for psychotic disorders. Whether youth with other diagnoses are experiencing prodromal symptoms of psychosis is unclear. It is likely that distressed parents and family members of children with severe behavior problems are willing to try antipsychotic medications as a last resort, lack of FDA approval notwithstanding. It is also possible that children with no mental health diagnosis whatsoever are being treated by clinicians who are unsure of what diagnosis to give or reluctant to give a diagnosis with high stigma. The differences in antipsychotic utilization rates are likely a combination of practice variation in diagnosing youth as well as in the propensity to prescribe these medications.

\section{Acknowledgments}

Research reported in this publication was supported by the National Institute of Mental Health (NIMH) (grant: U19MH092201). The content is solely the responsibility of the authors and does not necessarily represent the 
official views of the National Institutes of Health (NIH). Christine Stewart, Enid M. Hunkeler, Jeanne M. Madden, Rebecca C. Rossom, Christine Lu, Frances L. Lynch, Beth E. Waitzfelder, Karen A. Coleman, and Greg E. Simon have received research support from NIMH. John E. Zeber has received research support and support for travel from National Institutes of Health (NIH).

Jeanne M. Madden has received research support from NIH, FDA, and Agency for Healthcare Research and Quality.

Karen A. Coleman has received research support from NIMH, NIH, and Patient Centered Outcomes Research Institute.

Brian K. Ahmedani has received research support from NIMH.

John E. Zeber has received research support from VA Health Services Research \& Development, the Commonwealth Fund, and NIH.

Greg E. Simon has received research support from Bristol-Myers Squibb.

\section{References}

Recently published papers of particular interest have been highlighted as:

- Of importance

•• Of major importance

1. Olfson M, Crystal S, Huang C, Gerhard T. Trends in antipsychotic drug use by very young, privately insured children. J Am Acad Child Adolesc Psychiatry. 2010; 49(1):13-23. Epub 2010/03/11. [PubMed: 20215922]

*2. Olfson M, Blanco C, Liu SM, Wang S, Correll CU. National Trends in the Office-Based Treatment of Children, Adolescents, and Adults With Antipsychotics. Arch Gen Psychiatry. 2012:1-10. Epub 2012/08/08. Paper presents antipsychotic utilization estimates between 1993 and 2009 using nationally representative NAMCS data. Estimates are based on visits where a prescription occurred. Excellent discussion of reasons for practice variation in prescribing and increasing use over time.

*3. Andrade SE, Lo JC, Roblin D, Fouayzi H, Connor DF, Penfold RB, et al. Antipsychotic medication use among children and risk of diabetes mellitus. Pediatrics. 2011; 128(6):1135-41. Epub 2011/11/23. Study reports an almost fourfold increase in the rate of diabetes among youth using antipsychotic medications compared to youth using no psychotropic medications. [PubMed: 22106077]

*4. Correll CU. Safety and tolerability of antipsychotic treatment in young patients with schizophrenia. J Clin Psychiatry. 2011; 72(8):e26. Epub 2011/09/09. Seminal study on the side effects of antipsychotic medications in youth. [PubMed: 21899814]

*5. Maayan L, Correll CU. Weight gain and metabolic risks associated with antipsychotic medications in children and adolescents. J Child Adolesc Psychopharmacol. 2011; 21(6):517-35. Epub 2011/12/15. Review of 34 published head-to-head and placebo-controlled studies in youth with psychotic and bipolar disorders of the effects of antipsychotic medications on weight and metabolic disorders. Describes metabolic effects and number-needed-to-harm for individual agents. [PubMed: 22166172]

6. Cohen D, Bonnot O, Bodeau N, Consoli A, Laurent C. Adverse effects of second-generation antipsychotics in children and adolescents: a Bayesian meta-analysis. J Clin Psychopharmacol. 2012; 32(3):309-16. Epub 2012/05/01. [PubMed: 22544019]

*7. Seida JC, Schouten JR, Boylan K, Newton AS, Mousavi SS, Beaith A, et al. Antipsychotics for children and young adults: a comparative effectiveness review. Pediatrics. 2012; 129(3):e771-84. Epub 2012/02/22. Reviews the comparative effectiveness of individual agents for youth across 64 trials and 17 cohort studies. [PubMed: 22351885] 
8. Loy JH, Merry SN, Hetrick SE, Stasiak K. Atypical antipsychotics for disruptive behaviour disorders in children and youths. Cochrane Database Syst Rev. 2012; 9:CD008559. Epub 2012/09/14. [PubMed: 22972123]

*9. McKinney C, Renk K. Atypical antipsychotic medications in the management of disruptive behaviors in children: safety guidelines and recommendations. Clin Psychol Rev. 2011; 31(3): 465-71. Epub 2010/12/07. Paper discusses mechanisms of action and long-term outcomes for youth using antipsychotics. Good discussion of use for sedation rather than core reasons for disruptive behavior. [PubMed: 21130552]

10. Harrison JN, Cluxton-Keller F, Gross D. Antipsychotic medication prescribing trends in children and adolescents. J Pediatr Health Care. 2012; 26(2):139-45. Epub 2012/03/01. [PubMed: 22360933]

11. Maher AR, Maglione M, Bagley S, Suttorp M, Hu JH, Ewing B, et al. Efficacy and comparative effectiveness of atypical antipsychotic medications for off-label uses in adults: a systematic review and meta-analysis. JAMA. 2011; 306(12):1359-69. Epub 2011/09/29. [PubMed: 21954480]

12. Maher AR, Maglione M, Bagley S, Suttorp M, Hu JH, Ewing B, et al. Efficacy and Comparative Effectiveness of Atypical Antipsychotic Medications for Off-Label Uses in Adults. JAMA: The Journal of the American Medical Association. 2011; 306(12):1359-69. [PubMed: 21954480]

13. Penfold RB, Kelleher KJ, Wang W, Strange B, Pajer K. Pediatric uptake of a newly available antipsychotic medication. Pediatrics. 2010; 125(3):475-82. Epub 2010/02/10. [PubMed: 20142282]

*14. Alexander GC, Gallagher SA, Mascola A, Moloney RM, Stafford RS. Increasing off-label use of antipsychotic medications in the United States, 1995-2008. Pharmacoepidemiol Drug Saf. 2011; 20(2):177-84. Epub 2011/01/22. Only recent study estimating use from physician-reported prescribing. Categorizes the volume of off-label use by level of evidence. [PubMed: 21254289]

15. Hassler F, Reis O. Pharmacotherapy of disruptive behavior in mentally retarded subjects: A review of the current literature. Dev Disabil Res Rev. 2010; 16(3):265-72. Epub 2010/10/29. [PubMed: 20981765]

16. Aman MG, Gharabawi GM. Special Topic Advisory Panel on Transitioning to Risperidone Therapy in Patients With Mental R, Developmental D. Treatment of behavior disorders in mental retardation: report on transitioning to atypical antipsychotics, with an emphasis on risperidone. $\mathrm{J}$ Clin Psychiatry. 2004; 65(9):1197-210. Epub 2004/09/16. [PubMed: 15367046]

17. McDougle CJ, Stigler KA, Erickson CA, Posey DJ. Atypical antipsychotics in children and adolescents with autistic and other pervasive developmental disorders. J Clin Psychiatry. 2008; 69(Suppl 4):15-20. Epub 2008/06/27. [PubMed: 18533764]

18. Capone GT, Goyal P, Grados M, Smith B, Kammann H. Risperidone use in children with Down syndrome, severe intellectual disability, and comorbid autistic spectrum disorders: a naturalistic study. J Dev Behav Pediatr. 2008; 29(2):106-16. Epub 2008/03/20. [PubMed: 18349709]

*19. Crystal S, Olfson M, Huang C, Pincus H, Gerhard T. Broadened use of atypical antipsychotics: safety, effectiveness, and policy challenges. Health Aff (Millwood). 2009; 28(5):w770-81. Epub 2009/07/23. Compares antipsychotic utilization in both Medicaid (public) and MarketScan (commercial) data. [PubMed: 19622537]

*20. Dosreis S, Yoon Y, Rubin DM, Riddle MA, Noll E, Rothbard A. Antipsychotic treatment among youth in foster care. Pediatrics. 2011; 128(6):e1459-66. Epub 2011/11/23. Compares utilization among youth eligible for Medicaid on the basis of disability or low income to youth in foster care. [PubMed: 22106072]

**21. Matone M, Localio R, Huang YS, Dosreis S, Feudtner C, Rubin D. The Relationship between Mental Health Diagnosis and Treatment with Second-Generation Antipsychotics over Time: A National Study of U.S. Medicaid-Enrolled Children. Health Serv Res. 2012; 47(5):1836-60. Epub 2012/09/06. Comprehensive presentation of utilization rates in youth across 50 states and 6 years. Detailed rates available for youth 0-5, 6-11, and 12-18. [PubMed: 22946905]

22. Ellis WR, Huebner C, Vander Stoep A, Williams MA. Washington State exhibits wide regional variation in proportion of Medicaid-eligible children who get needed mental health care. Health Aff (Millwood). 2012; 31(5):990-9. Epub 2012/05/09. [PubMed: 22566438] 
23. Cummings JR, Wen H, Ko M, Druss BG. Geography and the Medicaid Mental Health Care Infrastructure: Implications for Health Care Reform. JAMA Psychiatry. 2013; 70(10):1084-90. Epub 2013/08/24. [PubMed: 23965816]

24. Harris E, Sorbero M, Kogan JN, Schuster J, Stein BD. Concurrent mental health therapy among medicaid-enrolled youths starting antipsychotic medications. Psychiatr Serv. 2012; 63(4):351-6. Epub 2012/02/18. [PubMed: 22337007]

25. Sturm R, Ringel JS, Andreyeva T. Geographic Disparities in Children's Mental Health Care. Pediatrics. 2003; 112(4):e308. [PubMed: 14523217]

26. Merikangas KR, He JP, Rapoport J, Vitiello B, Olfson M. Medication use in US youth with mental disorders. JAMA Pediatr. 2013; 167(2):141-8. Epub 2013/02/14. [PubMed: 23403911]

*27. Olfson M, He JP, Merikangas KR. Psychotropic medication treatment of adolescents: results from the national comorbidity survey-adolescent supplement. J Am Acad Child Adolesc Psychiatry. 2013; 52(4):378-88. Epub 2013/04/16. Estimates rates of antipsychotic use in youth across socio-demographic groups. Diagnoses were obtained using a validated instrument rather than administrative codes. [PubMed: 23582869]

28. Simon GE, Rutter CM, Stewart C, Pabiniak C, Wehnes L. Response to past depression treatments is not accurately recalled: comparison of structured recall and patient health questionnaire scores in medical records. J Clin Psychiatry. 2012; 73(12):1503-8. Epub 2013/01/08. [PubMed: 23290322]

29. Solomon DH, Stedman M, Licari A, Weinblatt ME, Maher N, Shadick N. Agreement between patient report and medical record review for medications used for rheumatoid arthritis: the accuracy of self-reported medication information in patient registries. Arthritis Rheum. 2007; 57(2):234-9. Epub 2007/03/03. [PubMed: 17330299]

30. Boudreau DM, Daling JR, Malone KE, Gardner JS, Blough DK, Heckbert SR. A validation study of patient interview data and pharmacy records for antihypertensive, statin, and antidepressant medication use among older women. Am J Epidemiol. 2004; 159(3):308-17. Epub 2004/01/27. [PubMed: 14742292]

31. van Gelder MM, van Rooij IA, de Walle HE, Roeleveld N, Bakker MK. Maternal recall of prescription medication use during pregnancy using a paper-based questionnaire: a validation study in the Netherlands. Drug Saf. 2013; 36(1):43-54. Epub 2013/01/15. [PubMed: 23315295]

32. West SL, Savitz DA, Koch G, Strom BL, Guess HA, Hartzema A. Recall accuracy for prescription medications: self-report compared with database information. Am J Epidemiol. 1995; 142(10): 1103-12. Epub 1995/11/15. [PubMed: 7485055] 


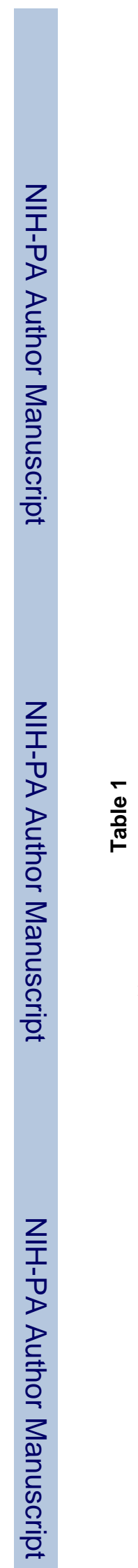

Penfold et al.

Page 14

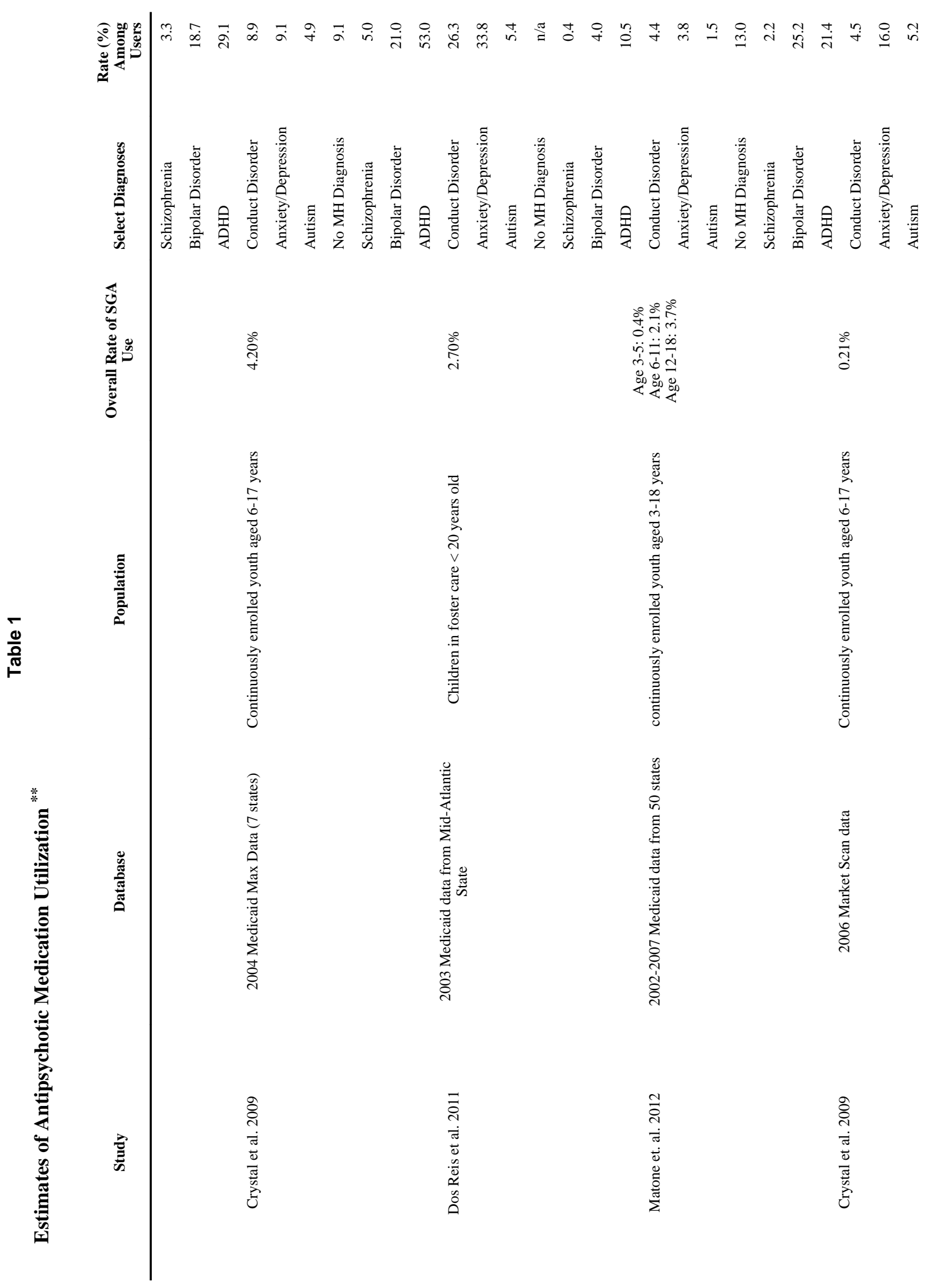

Curr Psychiatry Rep. Author manuscript; available in PMC 2014 December 01. 


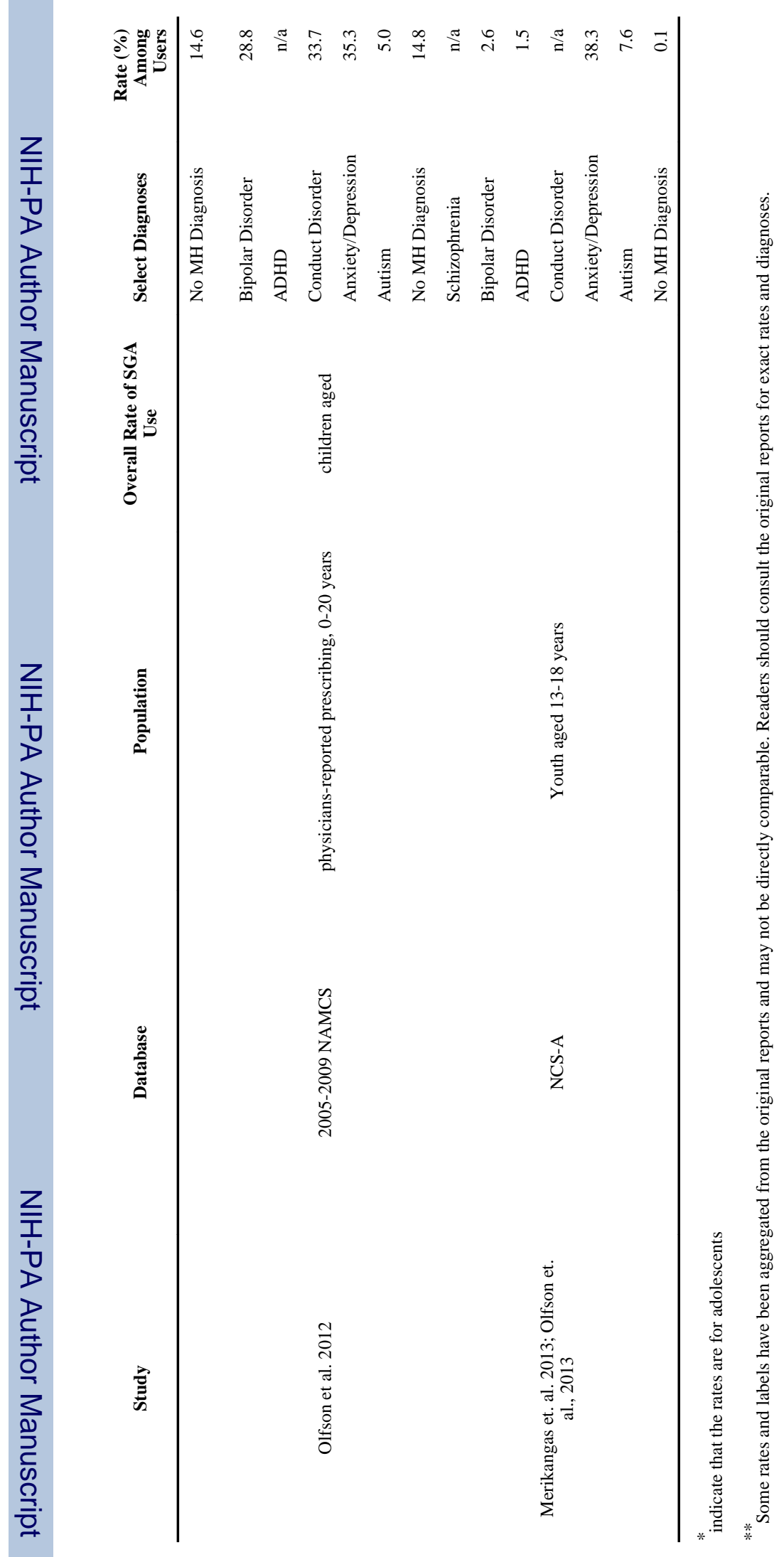

Curr Psychiatry Rep. Author manuscript; available in PMC 2014 December 01. 


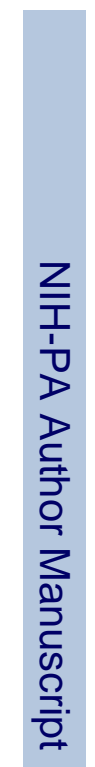

Penfold et al.

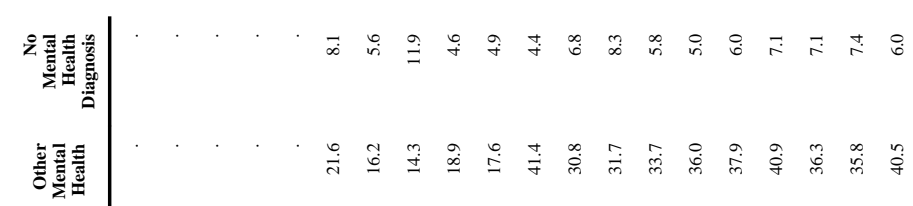

焉

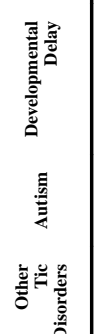

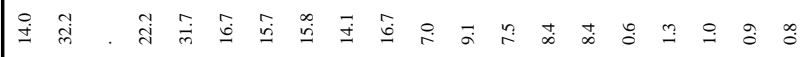

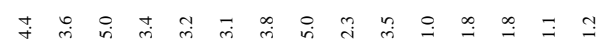

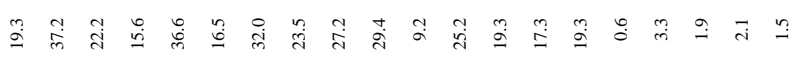

竧畜

臂量

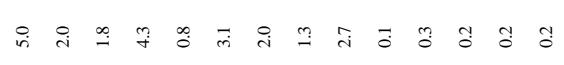

3 구의

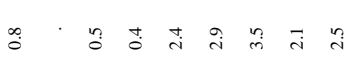

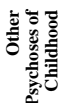

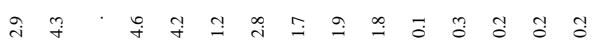

譬

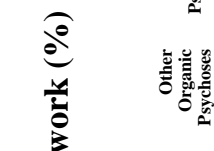

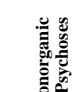

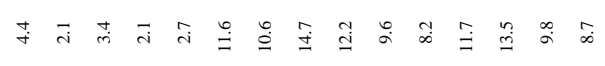

t.

ल

.

(1)

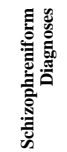

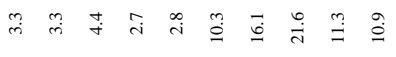

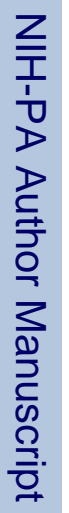

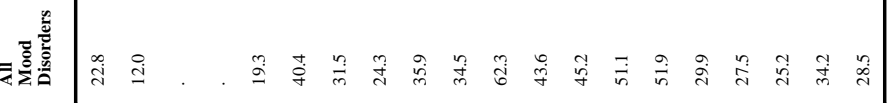

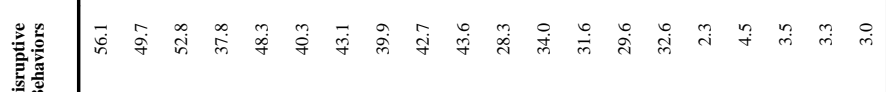

章 